\title{
Aspectos da regulação em saúde na visão de equipes de saúde da família de um município de pequeno porte
}

\author{
Aspects of healthcare regulations according to family health teams in a small city \\ Aspectos de la regulación de la salud en la visión de los equipos de salud de la familia de una \\ ciudad pequeña
}

Janise Braga Barros Ferreira, Daniela Cacilda Caldas, Luciane Loures dos Santos, Luciana Cisoto Ribeiro, Lucieli Dias

Pedreschi Chaves

\section{Resumo}

Objetivos: Identificar a visão de equipes da Estratégia Saúde da Família acerca do processo de regulação em saúde e caracterizar seus instrumentos quanto ao conteúdo informacional e sua utilização pela equipe. Métodos: Estudo de caráter descritivo, quantitativo e apoiado em fontes primária e secundária de dados, realizado em um município de pequeno porte. Foi aplicado um questionário aos profissionais de equipes de saúde da família, em 2012, com conteúdo referente ao processo de regulação em saúde e analisadas características de completude e legibilidade de informações registradas nas guias de referência às especialidades médicas. Resultados: Os profissionais da saúde da família relataram incertezas sobre a proposta conceitual e operativa do sistema de regulação em saúde. Apenas $50 \%$ dos participantes conheciam todos os serviços especializados de referência da rede de saúde. Cerca de $70 \%$ informaram a existência de protocolos na rede e/ou nas unidades de saúde. Houve prejuízo na completude e na legibilidade de informações das guias de referência. Conclusão: Os achados da pesquisa ratificaram a estreita relação entre o processo regulatório assistencial e a gestão da informação, bem como a importância da participação do profissional da saúde da família no mecanismo de regulação em saúde.

\section{Abstract}

Objectives: To identify the opinion of Family Health Strategy teams about the healthcare regulation process and describe their instruments concerning the informational content and its use by the team. Methods: A descriptive, quantitative study, supported by primary and secondary data sources and held in a small city. A questionnaire was administered to professionals of family health teams in 2012, with content referring to the regulation process in healthcare and completeness characteristics and readability of the information recorded in charts to medical specialties were analyzed. Results: The family health professionals reported uncertainty about the conceptual and operational proposal of the healthcare regulation system. Only $50 \%$ of the subjects knew about all the reference specialized services in the healthcare system. About $70 \%$ reported the existence of protocols in the network and/or in healthcare facilities. There were problems in the completeness and readability of information in medical charts. Conclusion: The research findings have ratified the close relationship between the healthcare regulatory process and information management, as well as the importance of the participation of family health professionals in the regulation mechanism in healthcare.

Como citar: Ferreira JBB, Caldas DC, Santos LL, Ribeiro LC, Chaves LDP. Aspectos da regulação em saúde na visão de equipes de saúde da família de um município de pequeno porte. Rev Bras Med Fam Comunidade. 2016;11(38):1-12. http://dx.doi.org/10.5712/rbmfc11(38)1188

\section{Palavras-chave:} Regulação e Fiscalização em Saúde Gestão da Informação Estratégia Saúde da Família Cidades Pequenas Protocolos Clínicos

Keywords: Health Care Coordination and Monitoring Information management Family Health Strategy Small cities Clinical protocols

Fonte de financiamento: declaram não haver. Parecer CEP: 454/CEP-CSE-FMRP-USP (FMRP-USP), aprovado em 12/08/2011 Conflito de interesses: declaram não haver. Procedência e revisão por pares: revisado por pares. Recebido em: 05/08/2015. Aprovado em: 16/11/2016. 


\section{Resumen}

Objetivos: Identificar la visión de equipos de la Estrategia de Salud Familiar acerca del proceso de regulación en salud y caracterizar sus instrumentos con respecto al contenido informativo y su uso por parte del equipo. Métodos: Estudio descriptivo, cuantitativo, basado en fuentes de datos primarios y secundarios, realizado en una ciudad pequeña. Se aplicó un cuestionario a los profesionales de los equipos de la estrategia de salud familiar en el 2012, con contenidos relacionados con el proceso de regulación en la salud y se analizó la integridad de las características y la legibilidad de la información registrada en las guías de referencia a las especialidades médicas. Resultados: Los profesionales de la Estrategia de Salud Familiar informaron incertidumbres acerca de la propuesta conceptual y del procedimiento funcional del sistema de regulación en salud. Sólo el $50 \%$ de los participantes conocía todos los servicios especializados de la red de salud. Aproximadamente el $70 \%$ informó la existencia de protocolos en la red y/o en los centros de salud. Hubo daños en la plenitud y legibilidad de la información de las guías de referencia. Conclusión: Los hallazgos de la investigación han ratificado la estrecha relación entre el proceso de la regulación asistencial y la gestión de la información, y la importancia de la participación del profesional de la estrategia de salud familiar en el mecanismo de regulación en salud.
Palabras clave:

Regulación y

Fiscalización en Salud

Gestión de la Información

Estrategia de Salud Familiar

Ciudades Pequeñas

Protocolos Clínicos

\section{Introdução}

Os países desenvolvidos, ao discutirem o desempenho dos sistemas de saúde e diferentes possibilidades organizacionais, enfatizaram os modelos centrados na Atenção Primária à Saúde (APS) influenciados pela Conferência de Alma Ata e isso refletiu no Brasil. ${ }^{1}$

Com o Sistema Único de Saúde (SUS), reforça-se o entendimento de que o mais importante para melhorar a condição de vida da população não é a assistência individual focada na cura, mas sim na promoção da qualidade de vida enfatizando a saúde e não a doença. ${ }^{2}$

Entretanto, em função da descentralização do SUS, muitos municípios possuem dificuldades para atingir as metas da APS, entre outros motivos, pelo fato de haver uma pequena capacitação para a gestão da saúde, pela falta de recursos financeiros e/ou ineficiência em sua aplicação, bem como indefinição do modelo de atenção à saúde que se deseja implantar, refletido na cultura curativista arraigada nos serviços de saúde. ${ }^{1}$

Neste sentido, o Governo Federal vem adotando ações para fortalecer a APS. Em 1994, o Ministério da Saúde (MS) lançou o Programa Saúde da Família (PSF), reconhecido como "um marco na incorporação da estratégia de atenção primária na política de saúde brasileira". ${ }^{3}$ Inicialmente, o PSF foi criado para ampliar a cobertura da APS nas áreas de risco, porém, por seus resultados satisfatórios, adquiriu centralidade na política pública de saúde. ${ }^{1}$

A Saúde da Família deixou de ser programa para se tornar a Estratégia da Saúde da Família (ESF) em 2006, traduzindo-se em um mecanismo para fortalecer o sistema de saúde, tornando-se o contato preferencial do usuário com o SUS. ${ }^{4}$

AESF, em sintonia com a APS, possui diretrizes organizativas fundamentais: conhecer as famílias do seu território para identificar os problemas de saúde mais prevalentes e as situações de risco existentes na comunidade; incentivar a comunidade a participar de ações de prevenção e de promoção à saúde; ofertar assistência integral às famílias e incentivar a corresponsabilização no cuidado à saúde junto à comunidade. ${ }^{3}$

No que diz respeito à coordenação do cuidado quanto ao acesso aos pontos da rede de saúde, por meio da referenciamento, destaca-se a potência da ação regulatória da APS. ${ }^{5}$ Esta ação na Rede de Atenção à Saúde (RAS) é essencial à continuidade da atenção prestada à população, e sem ela fica difícil 
se atingir a integralidade. Nos casos eletivos, esta ação, preferencialmente, se inicia na APS/ESF. Assim, a ESF necessita integrar a RAS, interligando-se aos seus demais pontos e com eles estabelecendo uma comunicação/articulação efetiva, lançando mão de vários instrumentos, dentre os quais destacam-se os protocolos clínicos e regulatórios. Entende-se por protocolos clínicos aqueles que descrevem a prática da assistência à saúde, baseada em evidências para subsidiar as decisões terapêuticas, enquanto que os protocolos regulatórios são instrumentos de ordenação dos fluxos. ${ }^{6}$

O papel da equipe da ESF é muito importante para a regulação do acesso, pois é ela que vai, na maioria das vezes, direcionar o usuário na RAS, determinando inclusive a utilização dos recursos humanos e tecnológicos, o que exige referências responsáveis e elucidativas. Assim, a expansão da ESF tem propiciado o maior exercício da ação regulatória pela atenção primária e pela gestão municipal. ${ }^{2}$

Por outro lado, se não houver um sistema de referência e contrarreferência* a contento que permita o encaminhamento recíproco dos pacientes entre os diferentes pontos da rede de saúde, a continuidade do atendimento na APS pode ficar comprometida, com repercussão desfavorável tanto para o paciente quanto para o sistema de saúde. Quando se particulariza a situação de saúde dos municípios de pequeno porte, a ação regulatória torna-se ainda mais necessária, pela dependência de recursos financeiros e humanos das demais esferas governamentais e porque a maioria desses territórios não possui todos os serviços, ou seja, carecem do bom funcionamento da RAS para a garantia da integralidade do acesso às ações de saúde. ${ }^{1}$

Do ponto de vista informacional, percebe-se a estreita ligação da ação regulatória com a qualidade da informação em saúde. Tem-se nos conteúdos informacionais dos instrumentos de regulação a possibilidade desta ação ser fundamentada nos princípios basilares do SUS, a integralidade, a equidade e a humanização.

É preciso registrar que o conteúdo informacional das guias de referência (GR), de contrarreferência (GCR) e dos protocolos pode ser considerado a matéria-prima da ação regulatória. ${ }^{6}$ É com base nas informações registradas nesses documentos que se procede à análise dos problemas de saúde e se decide qual a melhor opção terapêutica para cada caso, em função da priorização de risco/necessidade ${ }^{5}$ Tem-se clareada a importância destes instrumentos na regulação e, consequentemente, o papel das equipes de saúde responsáveis pela elaboração dos mesmos para que esse processo seja completo e resolutivo.

Neste momento, põe-se em evidência a potencial contribuição da Ciência da Informação $(\mathrm{Cl})$ para todo o processo regulatório agregando qualidade, principalmente, por deter conhecimentos acerca da gestão informacional e documental. ${ }^{7} \mathrm{~A} \mathrm{Cl}$ é o campo do conhecimento que se ocupa em investigar as propriedades e o comportamento da informação, a coordenação de seus fluxos e processamento, com a finalidade de otimização de sua acessibilidade e emprego. ${ }^{8}$

A viabilização de todos os processos pelos quais a informação precisa percorrer para ser interpretada e utilizada denota claramente a magnitude da $\mathrm{Cl}$ e, mais especificamente, sua contribuição para o processo de tomada de decisão no setor saúde. É certo que a gestão adequada das informações em saúde, no SUS, além de proporcionar um melhor atendimento clínico do usuário, também possibilita o acesso mais organizado e oportuno aos pontos da rede atenção, favorecendo a utilização eficiente dos recursos disponíveis. 
Destarte, o estudo da problemática regulatória focado, principalmente, na atuação das equipes da saúde da família suscita inquietações sobre o seu conhecimento a respeito desta temática, na medida em que se considera imprescindível o desempenho do profissional da atenção primária como agente regulador.

A equipe da ESF é quem direciona, na maioria das vezes, o usuário para os demais pontos da rede de atenção, influenciando diretamente na utilização de recursos humanos e tecnológicos, o que requer referências responsáveis e elucidativas. Deste modo, surge o questionamento de qual seria a percepção da equipe de saúde da família sobre o mecanismo de regulação em saúde, considerando o cenário de um município de pequeno porte e a rede de atenção à saúde local e regional.

Trata-se de temática pouco discutida nos âmbitos do SUS e acadêmico, o que pode ser constatado pela pequena produção técnica e científica disponível. ${ }^{9}$ A relevância deste estudo também se reflete no fato de sua proposição analítica fundar-se na perspectiva da Saúde Pública e da Cl, ou seja, um estudo baseado na interdisciplinaridade ensejando aumentar as possibilidades de compreensão do objeto investigado, na medida em que integra conhecimentos destas áreas.

O estudo teve como objetivos identificar a visão das equipes da ESF acerca do processo de regulação do acesso assistencial e caracterizar instrumentos de regulação em saúde quanto ao seu conteúdo informacional e utilização pela equipe da ESF.

\section{Métodos}

O estudo de caráter descritivo foi apoiado em fontes primárias e secundárias de dados, desenvolvido em Jardinópolis, SP, município de pequeno porte, classe II, ${ }^{* *}$ com população aproximada de 36.000 habitantes e densidade demográfica de 72,43 habitantes $/ \mathrm{km}^{2}$. Este município integra a Região de Saúde Aquífero Guarani, do Departamento Regional de Saúde XIII (DRS XIII) do estado de São Paulo. Do ponto de vista dos indicadores sociais, em 2010, o município apresentava: Índice Paulista de Responsabilidade Social (IPRS): Grupo 4 (quatro); Índice de Desenvolvimento Humano Municipal: 0,735 e Renda per capita (em reais correntes): $\mathrm{R} \$ 600,65 .^{10}$

A rede pública de saúde do município, no momento do estudo, era composta por um hospital de média complexidade e por nove Unidades de Saúde, cinco delas com a ESF. Participaram do estudo profissionais da ESF de quatro unidades de saúde de um total de cinco unidades. Os membros da equipe de saúde de uma unidade não participaram, pois no momento da coleta de dados a equipe estava em formação e não prestava atendimento à população.

Para o cumprimento dos objetivos da pesquisa, foi elaborado um instrumento, composto por 24 perguntas fechadas embasado, principalmente, na portaria ministerial que instituiu a Política Nacional de Regulação do SUS. ${ }^{11}$ As questões versavam sobre o conhecimento da proposta de regulação do SUS, o processo de regulação do acesso no município, o papel da ESF na regulação do acesso, o conhecimento dos serviços especializados de referência e dos instrumentos (protocolos clínicos e de regulação, GR e GCR) utilizados no processo de regulação assistencial, o número mensal de encaminhamentos e de agendamentos, a informatização das GR e a comunicação entre a equipe da ESF e a SMS (Quadro 1). 
Quadro 1. Instrumento de entrevista.

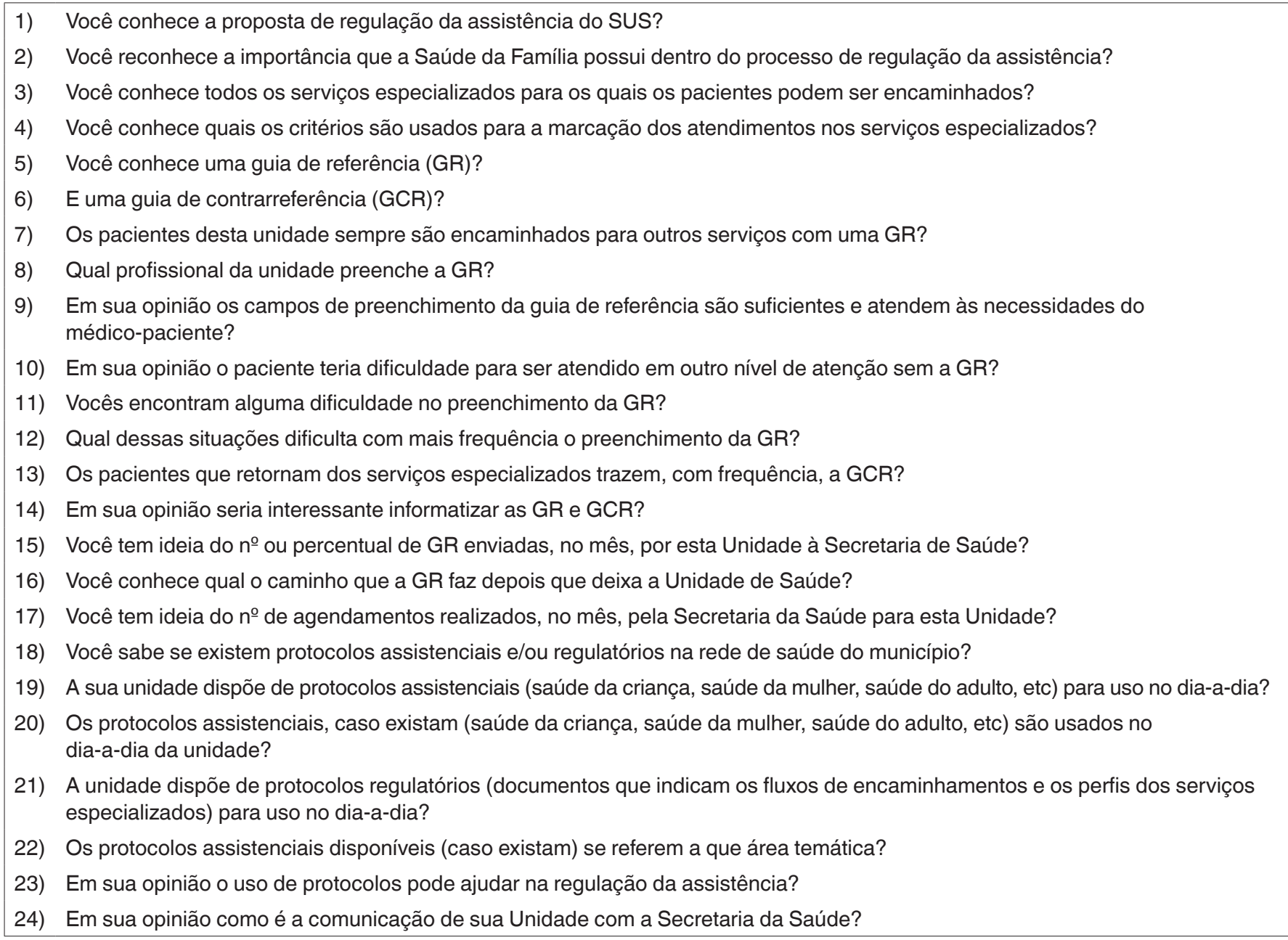

A coleta dos dados primários foi realizada por uma das pesquisadoras, no período de maio a junho de 2012. Importante pontuar que o pré-teste do instrumento foi realizado ${ }^{12}$ com a colaboração de três profissionais de saúde de outras unidades que não participaram do estudo, sendo executados pequenos ajustes semânticos na redação de algumas questões. Posteriormente, o instrumento de pesquisa foi aplicado, por meio de entrevista, aos profissionais das ESF, de maio a junho de 2012.

A regulação das ações de saúde, no município, tinha na GR, em suporte papel, seu principal documento para a continuidade da assistência. Assim, foi analisado o conteúdo informacional de 48 GR preenchidas pelas equipes da ESF e enviadas para o centro regulador da SMS, no mês de agosto de 2011. Optou-se por analisar o total das guias emitidas no mês de agosto, pelo fato de ser um mês típico, o que menos poderia interferir no agendamento rotineiro das consultas médicas. ${ }^{13}$ Interessante assinalar que, em média, o centro regulador da SMS recebia um total de 203 GR por mês, sendo em torno de $24 \%$ oriundos das equipes de saúde da família.

A análise comportou a observação da completude e legibilidade de oito variáveis: história pregressa e quadro clínico; diagnóstico ou hipótese diagnóstica; exames realizados, datas e resultados; conduta 
terapêutica; motivo principal do encaminhamento; nome do médico solicitante; inscrição no Conselho Regional de Medicina (CRM) e data da solicitação. Cabe esclarecer que a análise das GR foi realizada por uma das pesquisadoras.

$\mathrm{Na}$ análise da completude da informação (entendida como existência de registro) foi tabulada a presença ( $\operatorname{sim}$ ) ou ausência (não) do preenchimento dos campos da GR relacionados às variáveis selecionadas no estudo; na análise de legibilidade (considerada como clareza e nitidez caligráfica) a pesquisadora avaliava se o preenchimento dos campos relativos às variáveis estudadas encontrava-se legível (sim) ou não.

O banco de dados foi criado no programa Epilnfo, for Windows. Os dados primários e secundários foram analisados estatisticamente, contemplando valores absolutos e relativos. A pesquisa foi aprovada pelo Comitê de Ética em Pesquisa do Centro de Saúde Escola da Faculdade de Medicina de Ribeirão Preto-USP (protocolo no 454; CAEE:0014.0.175.000-11).

\section{Resultados}

Dos 40 profissionais de saúde das 4 unidades de saúde, 38 (95\%) participaram da pesquisa, dos quais 3 eram médicos generalistas, 4 enfermeiros, 8 técnicos em enfermagem e 23 agentes comunitários de saúde (ACS). No momento da coleta dos dados, duas equipes estavam incompletas (uma sem um ACS e outra sem o médico generalista). A média de idade dos participantes foi de 29,6 anos.

Todos os participantes reconheceram a importância da ESF no processo de regulação do acesso, dos quais $92,1 \%$ afirmaram conhecer sua fundamentação teórica, 94,7\% os serviços especializados de referência da RAS e mais da metade dos entrevistados $(65,8 \%)$ reconheceram a comunicação da ESF com a sede da SMS como ótima/boa.

Quanto à existência dos instrumentos de regulação, cerca de $70 \%$ informaram que existiam protocolos clínicos e/ou regulatórios na RAS e 74\% em sua própria unidade de saúde, sendo os mais referidos os da área de saúde da mulher (76,3\%), da criança (50\%) e adulto e idoso $(47,4 \%)$.

Quanto à utilização, 68,4\% afirmaram que os protocolos clínicos e os de regulação eram empregados pelos profissionais. A GR foi identificada pela maioria dos profissionais $(97,4 \%)$, que apontaram sua importância para a continuidade da assistência. Eles afirmaram (79\%) conhecer o fluxo operacional deste instrumento e 95\% referiram que sem ela os usuários não conseguiriam atendimento em outros pontos da RAS.

Quanto à GCR, apesar de ser conhecida pelas equipes (76,3\%), era pouco utilizada pelos serviços da RAS. Mais da metade $(52,6 \%)$ referiu que os pacientes retornavam do atendimento especializado sem este documento. Ainda, segundo $95 \%$ dos entrevistados seria fundamental a informatização do processo de emissão das GR e GCR.

Quanto à emissão das GR pela unidade de saúde da família, 86,8\% dos profissionais afirmaram que não tinham conhecimento sobre o total de guias emitidas por mês, nem sobre o total de agendamentos mensais, realizados pela SMS, de sua unidade de saúde nos serviços de saúde especializados. Apenas um terço dos médicos e $25 \%$ dos enfermeiros tinham conhecimento sobre o número de agendamentos mensais. 
Os participantes informaram que os principais profissionais que preenchiam as GR eram os médicos $(89,5 \%)$ e enfermeiros $(31,6 \%)$, mas que outros profissionais também poderiam preenchê-la. A maioria dos profissionais $(68,4 \%)$ informou que os campos da GR eram suficientes e atendiam às necessidade do atendimento, e 55,3\% que não apresentavam dificuldades em seu preenchimento. Quando se analisa esta resposta, selecionando somente os 7 profissionais que mais preenchiam essa ferramenta (médicos e enfermeiros), $57,1 \%$ (4) afirmaram que os campos de preenchimentos eram suficientes. Em relação ao preenchimento da GR $44,7 \%$ dos profissionais informaram que o preenchimento manual (qualidade da letra) era o principal fator dificultador.

A análise das 48 GR mostrou que a completude das informações atingiu percentuais superiores a $75 \%$, em 6 das 8 variáveis estudadas. A completude das informações atingiu o percentual de $100 \%$ somente nas variáveis história pregressa e quadro clínico. A variável conduta terapêutica foi a que registrou o menor percentual de completude (31,25\%). A variável legibilidade apresentou percentuais inferiores a $70 \%$ em cinco dos oito critérios avaliados.

Os menores percentuais de legibilidade da informação foram registrados nas variáveis: conduta terapêutica $(18,75 \%)$, exames realizados (31,25\%), diagnóstico e hipótese diagnóstica $(47,91 \%)$ e história pregressa e quadro clínico (50\%). Em nenhuma das variáveis analisadas observou-se $100 \%$ de legibilidade. Em 29,2\% das GR analisadas não foi possível identificar a especialidade médica solicitada pela ilegibilidade do registro da informação (Tabela 1).

Tabela 1. Análise do conteúdo informacional das GR, segundo completude e legibilidade dos registros, Jardinópolis, agosto de 2012.

\begin{tabular}{|c|c|c|c|c|}
\hline \multirow{2}{*}{ Variável } & \multicolumn{2}{|c|}{ Completude } & \multicolumn{2}{|c|}{ Legibilidade } \\
\hline & $\mathbf{N}$ & $\%$ & $\mathbf{N}$ & $\%$ \\
\hline História pregressa e quadro clínico & 48 & 100,0 & 24 & 50,0 \\
\hline Diagnóstico e hipótese diagnóstica & 44 & 91,7 & 23 & 47,9 \\
\hline Exames realizados e datas & 38 & 79,2 & 15 & 31,3 \\
\hline Conduta terapêutica & 15 & 31,3 & 09 & 18,8 \\
\hline Motivo do encaminhamento & 34 & 70,8 & 31 & 55,6 \\
\hline Nome do médico & 47 & 97,9 & 35 & 72,9 \\
\hline CRM & 43 & 89,6 & 42 & 87,5 \\
\hline Data da solicitação & 47 & 97,9 & 46 & 97,9 \\
\hline
\end{tabular}

Fonte: Elaboração própria

\section{Discussão}

A regulação no SUS é uma importante temática discutida mais efetivamente nos últimos 15 anos. ${ }^{11}$ A importância de se promover a discussão sobre regulação junto aos profissionais da saúde reside no fato da necessidade de se identificar as fortalezas e desafios a serem ultrapassados no desenvolvimento desta ação de saúde. ${ }^{14}$

Os profissionais da saúde da família que participaram do estudo relataram incertezas sobre a proposta conceitual e operativa do atual sistema de regulação do SUS. É essencial que os profissionais da APS, centro da coordenação do cuidado, reconheçam o seu papel na regulação, principalmente em municípios de pequeno porte, dependentes desta ação para acesso aos demais pontos da RAS. 
Apenas 50\% dos participantes conheciam todos os serviços especializados de referência da RAS, dado intrigante, diante da premissa de que APS é responsável por coordenar o cuidado. O conhecimento acerca dos serviços disponíveis na RAS e a comunicação entre eles permite a aproximação entre os profissionais, favorecendo a busca de soluções conjuntas para a condução e resolução dos casos. ${ }^{5,15}$

Ferreira et al. ${ }^{5}$ afirmam que a RAS deve ser "produtora de cuidados, de forma compartilhada, progressiva, humanizada, resolutiva e regionalizada e atenta para a garantia da equidade e da integralidade. Uma rede solidária."

Quanto aos protocolos clínicos e regulatórios, os participantes os identificaram como ferramentas valiosas para o exercício das ações clínicas e de regulação, na perspectiva da integralidade e da continuidade da atenção, embora apenas $68 \%$ tenham informado o seu uso pelas equipes.

Com o auxílio desses instrumentos, é possível estabelecer em qual ponto da RAS o usuário terá melhor atendido o seu problema, pela análise do perfil assistencial da unidade executante e do fluxo mais indicado para os referenciamentos. Por meio dessas análises, que envolvem a gestão das informações em saúde no processo regulatório, pode-se ter o aumento de referências mais oportunas e responsáveis ${ }^{6}$. Porém, é necessário que as equipes da ESF tenham o cuidado em usá-los com parcimônia, não tornando o atendimento "engessado" ou "mecânico". ${ }^{16}$

No modelo de rede regulada do município é necessário que toda a equipe discuta os instrumentoschave para o desenvolvimento da assistência integral ao usuário. Assim, como no município estudado, outros também utilizam a GR como instrumento de regulação, ratificando sua representatividade para o desenvolvimento da ação regulatória no SUS. ${ }^{6}$

Assinala-se que o processo de regulação dos casos eletivos, em sua grande maioria, tem seu início na APS/SF quando o usuário acessa o sistema, espontaneamente ou não, por esta porta de entrada. A SMS do município estudado era responsável pela recepção dos referenciamentos, por meio de um centro regulador que processava as referências e indicava os serviços especializados, devendo realizar o cotejamento das necessidades do usuário com o perfil dos estabelecimentos de saúde da RAS. Quando há uma insuficiência de informações nos instrumentos regulatórios, como o observado neste estudo pela incompletude e ilegibilidade de dados, a regulação sofre prejuízo.

A depender do modo de operacionalização do centro regulador pode-se contatar o responsável pelo referenciamento para que sejam complementadas as informações. Da mesma forma, compete ao centro regulador da SMS informar às unidades solicitantes sobre o desfecho dado aos casos referenciados. Este procedimento fortalece o processo de regulação do acesso na medida em que integra os pontos da RAS e permite a busca de soluções conjuntas.

No entanto, Santos ${ }^{17}$ comenta que esta "integração fundamenta-se na modificação das práticas profissionais e na cooperação entre os diferentes serviços que compõem a rede assistencial". É uma rede de trocas, que certamente promoverá a continuidade da atenção à saúde.

Do mesmo modo, a GCR tem sua relevância neste processo, pois deve conter o histórico do atendimento do usuário, além das devidas orientações para o seguimento do caso pela equipe da ESF e o seu não preenchimento pode representar a perda ou a dificuldade de acesso a um conjunto informacional diretamente relacionado à coordenação do cuidado. No entanto, a realidade da prática assistencial aponta que a contrarreferência é negligenciada na RAS. ${ }^{5}$ Desta forma, torna-se evidente que a coordenação 
do cuidado depende da integração da RAS, relacionando-se a um robusto sistema de referência e contrarreferência. ${ }^{18}$

Ainda que a informatização da regulação tenha sido apontada como necessidade para 95\% dos entrevistados, em municípios de pequeno porte essa realidade é muito incipiente e a troca de informações ocorre, na maioria das vezes, por meio de contato telefônico ou registro em suporte papel. Assim, foi grande o apelo para a informatização do processo regulatório, pois as experiências de sucesso remetem à investimentos em tecnologias de informação e comunicação (TIC), na implantação do sistema de regulação. ${ }^{19,20}$

Com a informatização, o conhecimento sobre o processo regulatório realizado pela ESF e, consequentemente, a condução da atenção a sua população são facilitados. Esse saber auxilia na qualificação da assistência prestada e permite a reflexão das equipes sobre o seu processo de trabalho, em relação à capacidade resolutiva, a organização e operacionalização da ação regulatória.

Merece destaque o fato de os participantes da pesquisa não utilizarem informações por eles produzidas e cuja análise e interpretação poderiam contribuir com a ação regulatória desenvolvida por eles e se traduzir em substrato para o planejamento de novas ações de saúde, influenciando a organização da oferta assistencial na RAS.

Os resultados do estudo apontaram que, além do médico, outros profissionais da equipe preenchiam a GR. Ressalta-se que este é um instrumento de cunho sigiloso, cujas informações pertencem ao usuário, devendo ser tratadas com respeito e ética. É de extrema importância que os profissionais que a utilizam tenham o cuidado com o preenchimento completo e legível, com o seu manuseio e armazenamento. ${ }^{21}$

Assim como qualquer outro documento, a GR contém registros importantes que devem ser organizados, preservados e acessíveis para o desenvolvimento do processo regulatório. Entretanto, o problema da gestão documental é um dilema de muitas instituições públicas, inclusive as de saúde. ${ }^{7}$

O fato de a GR, no sistema de saúde estudado, ser em suporte papel favorece o surgimento de registros ilegíveis ou incompletos, o que foi observado quando se analisou o conteúdo informacional das GR. Nota-se aqui um paradoxo, pois os participantes reconheceram a importância deste instrumento para a regulação do acesso e em contrapartida tanto a completude quanto a legibilidade estavam comprometidas nas guias analisadas. A qualidade do registro em saúde tem relação direta com a ação regulatória e com o desfecho das respostas aos problemas de saúde. Desta forma, não só a letra ilegível, como também a incompletude dos dados, dificulta o trabalho dos reguladores e a resolutividade das ações de saúde e dos serviços. ${ }^{5}$

Neste sentido, a gestão da informação contribui com o processo regulatório, na medida em que favorece a organização dos dados, o registro das informações e auxilia a tomada de decisão, aumentando a possibilidade de acerto. ${ }^{22}$

É certo que cada categoria profissional se envolve de alguma maneira com o processo regulatório, o que exige a atuação multiprofissional integrada. Logo, a apropriação pela equipe de saúde do conjunto informacional envolvido no processo regulatório tem implicações específicas dentro do processo de trabalho da saúde da família e, ainda, na totalidade do movimento regulatório, com reflexos no resultado da assistência, aflorando a importância da competência informacional para melhorar o desempenho desta ação. ${ }^{23}$ 
Os achados da pesquisa ratificaram a estreita relação entre a gestão da informação e o processo regulatório assistencial e estimulam a reflexão sobre a importância de se compreender a concepção do processo regulatório e a participação de cada profissional, neste processo, que tem na gestão da informação em saúde (produção, manuseio, guarda e recuperação, uso e disseminação) seu principal elemento constitutivo.

A qualidade do registro em saúde tem relação direta com a ação regulatória e se não houver um compromisso com a completude e legibilidade das informações registradas nos instrumentos regulatórios poderá haver um comprometimento desta ação, com repercussões insatisfatórias para o sistema de saúde e o usuário.

Em que pesem as limitações do estudo do ponto de vista metodológico que restrigem a generalização dos resultados e notando que a pesquisa não teve a pretensão de abarcar esta temática em toda a sua extensão e profundidade, acredita-se que os resultados encontrados contribuirão para uma melhor compreensão da realidade de um município de pequeno porte, quanto ao desenvolvimento do processo de regulação do acesso destacando a interface entre a assistência e a gestão da informação.

Com base nos achados da pesquisa, ressalta-se que os profissionais de saúde precisam adquirir novas habilidades para o desempenho de suas funções nos serviços de saúde, em um modelo de atenção centrado no usuário. Nesse sentido, o desenvolvimento de competência informacional para o exercício das ações regulatórias, por meio da educação permanente, apresenta-se como uma interessante estratégia a ser desenvolvida nos componentes assistenciais e gerenciais dos serviços de saúde, além da necessidade de investimento em novas tecnologias de informação e comunicação.

\section{Referências}

1. Castro ALB, Machado CV. A política de atenção primária à saúde no Brasil: notas sobre a regulação e o financiamento federal. Cad Saúde Pública. 2010;26(4):693-705. DOI: http://dx.doi.org/10.1590/S0102-311X2010000400012

2. Paim J, Travassos C, Almeida C, Bahia L, Macinko J. The Brazilian health system: history, advances, and challenges. Lancet. 2011;377(9779):1778-97. DOI: http://dx.doi.org/10.1016/S0140-6736(11)60054-8

3. Escorel S, Giovanella L, Mendonça MHM, Senna MCM. Programa de Saúde da Família e a construção de um novo modelo para a atenção básica no Brasil. Rev Panam Salud Publica. 2007;21(2-3):164-76. DOI: http://dx.doi.org/10.1590/S1020-49892007000200011

4. Brasil. Ministério da Saúde. Secretaria de Atenção à Saúde. Departamento de Atenção Básica. Política nacional de atenção básica. Brasília: Ministério da Saúde, 2007.

5. Ferreira JBB, Mishima SM, Santos JS, Forster AC, Ferraz CA. O complexo regulador da assistência à saúde na perspectiva de seus sujeitos operadores. Interface (Botucatu). 2010;14(33):345-58. DOI: http://dx.doi.org/10.1590/S1414-32832010000200009

6. Ferri SMN, Ferreira JBB, Almeida EF, Santos JS. Protocolos clínicos e de regulação: motivações para elaboração e uso. In: Santos JS, ed. Protocolos clínicos e de regulação: acesso à rede de saúde. Rio de Janeiro: Elsevier; 2012. p. 9-21.

7. Calderon WR, Cornelsen JM, Pavezi N, Lopes MA. O processo de gestão documental e da informação arquivística no ambiente universitário. Ci Inf. 2004;33(3):97-104. DOI: http://dx.doi.org/10.1590/S0100-19652004000300011

8. Saracevic T. Ciência da informação: origem, evolução e relações. Perspec Ci Inf. 1996;1(1):41-62.

9. Vilarins GCM, Shimizu HE, Gutierrez MMU. A regulação em saúde: aspectos conceituais e operacionais. Saúde Debate. 2012;36(95):640-7. DOI: http://dx.doi.org/10.1590/S0103-11042012000400016

10. Seade. Fundação Sistema Estadual de Análise de dados. Perfil municipal - Jardinópolis. [Internet]. 2011. [citado 2011 Abr 10] Disponível em: http://produtos.seade.gov.br/produtos/perfil/perfilMunEstado.php 
11. Brasil. Ministério da Saúde. Portaria ํo 1.559, 1ํ de agosto de 2008. Institui a Política Nacional de Regulação do Sistema Único de Saúde - SUS. Brasília: Diário Oficial da União; 2008.

12. Vergara SC. Métodos de coleta de dados no campo. São Paulo: Atlas; 2009.

13. Tanaka OY, Melo C. Avaliação de programa de saúde do adolescente: um modo de fazer. São Paulo: EDUSP; 2001.

14. Cotta RMM, Schott M, Azeredo CM, Franceschini SCC, Priore SE, Dias G. Organização do trabalho e perfil dos profissionais do Programa Saúde da Família: um desafio na reestruturação da atenção básica em saúde. Epidemiol Serv Saúde. 2006;15(3):7-18. DOI: http://dx.doi.org/10.5123/S1679-49742006000300002

15. Starfield B. Atenção primaria: equilíbrio entre necessidades de saúde, serviços e tecnologia. Brasília: UNESCO, Ministério da Saúde; 2004. 726 p.

16. Werneck MAF, Faria HP, Campos KFC. Protocolos de cuidados à saúde e de organização do serviço [internet]. Belo Horizonte:Nescon/UFMG, Coopmed, 2009. [citado 2012 Nov 19]. Disponível em: http://rumosaude.com.br/content/data/files/ files_contact_407/Guia\%20para\%20elabora\%C3\%A7\%C3\%A30\%20de\%20protocolos.pdf

17. Santos JS. Protocolo para acesso aos serviços do Sistema Único de Saúde. In: Santos JS, ed. Protocolos clínicos e de regulação: acesso à rede de saúde. Rio de Janeiro: Elsevier; 2012. p. 3-7.

18. Serra CG, Rodrigues PHA. Avaliação da referência e contrarreferência no Programa Saúde da Família na Região Metropolitana do Rio de Janeiro (RJ, Brasil). Ciênc Saúde Coletiva. 2010;15(Supl 3):3579-86. DOI: http://dx.doi.org/10.1590/S1413-81232010000900033

19. Giovanella L, Mendonça MHM, Almeida PF, Escorel S, Senna MCM, Fausto MCR, et al. Saúde da família: limites e possibilidades para uma abordagem integral de atenção primáriaà saúde no Brasil. Ciênc Saúde Coletiva. 2009;14(3):783-94. DOI: http://dx.doi.org/10.1590/S1413-81232009000300014

20. Rocha RV. O papel da tecnologia no contexto da gestão do conhecimento: um estudo de caso aplicado numa empresa varejista [mba]. Porto Alegre: Universidade do Vale dos Sinos; 2010. [citado 2012 Nov 20]. Disponível em: http://docplayer.com.br/529961-O-papel-da-tecnologia-da-informacao-no-contexto-da-gestao-do-conhecimento-umestudo-de-caso-aplicado-numa-empresa-varejista.html

21. Marchiori PZ. A ciência e a gestão da informação: compatibilidade no espaço profissional. Ci Inf. 2002;31(2):72-9. DOI: http://dx.doi.org/10.1590/S0100-19652002000200008

22. Guimarães MP, Évora YDM. Sistema de informação: instrumento para a tomada de decisão no exercício da gerencia. Ci Inf. 2004;33(1):72-80. DOI: http://dx.doi.org/10.1590/S0100-19652004000100009

23. Vitorino EV. Competência informacional: princípios para a formação contínua de profissionais da informação em bibliotecas universitárias. In: Anais do XV Seminário Nacional de Bibliotecas Universitárias [internet]; 2008 Nov 10-14; São Paulo, Brasil. [citado 2016 Dec 15]. Disponível em: http://www.sbu.unicamp.br/snbu2008/anais/site/pdfs/2698.pdf

* Contrarreferência: Ato formal de encaminhar um paciente ao estabelecimento de origem, após a resolução da causa responsável pela referência (Brasil. Tesauro Eletrônico, 2016).

** Classe II: municípios de 20.001 a 50.000 habitantes (IBGE, 2010). 
Janise Braga Barros Ferreira. Departamento de Medicina Social da Faculdade de Medicina de Ribeirão Preto, Universidade de São Paulo (FMRP-USP).

Ribeirão Preto, SP, Brasil. janise@fmrp.usp.br

Daniela Cacilda Caldas. Universidade de São Paulo (USP). Ribeirão Preto, SP, Brasil. dani_caldas08@ hotmail.com

Luciane Loures dos Santos. Departamento de Medicina Social da Faculdade de Medicina de Ribeirão Preto, Universidade de São Paulo (FMRP-USP). Ribeirão Preto, SP, Brasil. luloures@ @mrp.usp.br (Autora correspondente)

Luciana Cisoto Ribeiro. Departamento de Medicina Social da Faculdade de Medicina de Ribeirão Preto, Universidade de São Paulo (FMRP-USP). Ribeirão Preto, SP, Brasil. Icisoto@fmrp.usp.br

Lucieli Dias Pedreschi Chaves. Departamento de Enfermagem Geral e Especializada da Escola de Enfermagem de Ribeirão Preto da Universidade de São Paulo (EERP).

Ribeirão Preto, SP, Brasil. dpchaves@eerp.usp.br 